\title{
9. Menschlicher Umgang mit verwirrten Patienten
}

\author{
Eckhard Schlauß
}

\section{Der verwirrte Patient}

Der „verwirrte Patient“ - egal ob dement oder dement und delirant - bedarf einer empathischen Gestaltung der Begegnung. De facto ist jede Interaktion auch Kommunikation, und sollte bewusst eingesetzt werden. Das Gespräch mit einem „verwirrten Patienten“ hat immer auf Augenhöhe (gerade auch im wörtlichen Sinn!) zu erfolgen.

Was ist person-zentrierter Umgang - VIPS nach Dawn Brooker (2008, 17ff.)?

- V - Valorieren (wertschätzen) Sie Menschen mit Demenz und ihre Pflegepersonen.

- I-Behandeln Sie die Menschen als Individuen.

- P - Betrachten Sie die Welt aus der Perspektive von Menschen mit Demenz.

- S-Schaffen Sie eine positive soziale Umgebung, die Wohlbefinden ermöglicht.

Diese Regeln sollten die Grundlage „Ihrer Philosophie“ für jede Interaktion mit „verwirrten Patienten“ sein.

\section{Der demente vs. der verwirrte Patient}

An dieser Stelle muss eine Differenzierung erfolgen, da ein Delir sowohl ursächlich als auch symptomatisch zu behandeln ist. Bitte beachten Sie die folgenden charakteristischen Merkmale: 
- Er ist unaufmerksam - leicht ablenkbar.

- Er gibt unpassende Antworten.

- Er wechselt zwischen einem verwirrten und klaren Zustand.

- Er kann nicht verstehen, wo er ist.

- Er ist unruhig, ärgerlich, aggressiv oder auch teilnahmslos.

- Er hat Angst.

- Er kann Dinge sehen, hören oder riechen, die nicht da sind (Halluzinationen).

Führen Sie bitte einen kurzen Aufmerksamkeitstest durch - Monate rückwärts beginnend mit Dezember abfragen! Für Patienten, die eine Aphasie haben, oder denen aus anderen Gründen eine verbale Kommunikation nicht möglich ist, gibt es einen kurzen Aufmerksamkeitstest aus einer entsprechenden Testbatterie (vgl. Cieplinska-Legge et al. 2013). Im Folgenden wird der „delirante Patient“ nicht mehr speziell betrachtet.

\section{Herausforderndes Verhalten - beim dementen Patienten}

Infolge der gestörten Interaktion und Kommunikation zwischen neurologisch Behinderten (dementen Patienten) und Menschen, deren Kulturtechniken intakt sind, kommt es zu Missverständnissen und Störungen, in deren Folge Formen herausfordernden Verhaltens (fehlbeschrieben als Symptome!) seitens der Menschen mit Demenz entstehen, die zum eigentlichen „Problem“ werden. In einem Milieu mit einem hohen Maß an Expressivität und einem geringen Grad an Dominanz kann ungewöhnliches und bizarres Verhalten toleriert, begleitet, ermöglicht und „gehalten“ werden.

Der Umgang/die Kommunikation wird aus einer grundlegend therapeutischen, zulassenden Haltung heraus mit Wertschätzung, Echtheit und Empathie gestaltet. Es geht darum, den anderen einfühlend zu verstehen und ihn in der Welt seiner Szenen zu begleiten, nicht allein zu lassen, sondern - auch im Schreien auszuhalten, bis der Zustand sich ändert.

Menschen mit Demenz können nicht mehr „positiv bei sich selbst intervenieren“ bzw. sich durch Selbstinstruktionen aus belastenden Gefühlen befreien. Daher sollten Sie diese Menschen empathisch begleiten, mit ihren Gefühlen mitgehen und Ihnen helfen, diese Gefühle zu wandeln.

Der Umgang/die Kommunikation sollte offen und nicht durch vorab festgelegte Ablaufsysteme „geschlossen“ sein. In der Situation muss herausgefunden und erarbeitet werden, was jetzt und hier Sinn machen kann. Eine sich an der Lust und am Humor orientierende Suchhaltung mit genügend Neugierde und Phantasie ist nötig, um „Schlüssel“ zu den Welten und Szenen der Menschen mit Demenz zu gewinnen. 
Arbeiten Sie nicht gegen die „Symptome“. Vermeiden Sie diesen Fehler! Verstehen Sie alles herausfordernde Verhalten des demenziell erkrankten Menschen als Kommunikationsversuch.

Arbeit mit Menschen mit Demenz ist therapeutische Körperarbeit. Alle Menschen, die demenziell erkrankte Menschen betreuen, müssen lernen, schreiende Menschen zu halten, Trost zu vermitteln, zu streicheln, zu berühren, den eigenen Leib einzusetzen.

Dies wird ohne körpertherapeutische Selbsterfahrung nicht möglich sein.

Setzen Sie körperliche Nähe bewusst ein. Diese Selbsterfahrung sollte sehr bedacht und behutsam gestaltet werden.

Dies gilt in gleicher Art und Weise für den demenziell erkrankten Menschen und auch für Sie!

Sie stellen sich dabei mit Ihrer ganzen Person zur Verfügung und eben nicht als objektivierender, rationaler, distanziert-sachlicher Experte. Das zweckrationaltechnische Ideal des neutralen Experten ist als Widerstand gegen das Verstehen imaginärer Lebenswelten und als Zurückweisung des Beziehungsangebotes des Dementen zu analysieren (Gröning 1997). Sie verfügen über entsprechendes Fachwissen, aber der demenziell erkrankte Mensch (und nicht zu vergessen der betreuende Angehörige und/oder andere betreuende Personen) ist der Experte. Sie sollten sein Berater und Interessensvertreter sein.

\section{Hinweise zur Kommunikation}

Bei der Kommunikation mit demenziell erkrankten Menschen sind viele Probleme möglich. Folgende kommunikative Eigenheiten sind charakteristisch für den dementen Patienten:

- Er beginnt kein Gespräch bzw. beteiligt sich nicht daran.

- Er erzählt immer dasselbe.

- Er hat Wortfindungsprobleme.

- Er versteht keinen Spaß.

- Er lässt sich nicht überzeugen.

- Er versteht nicht, was man ihm sagt.

- Er behauptet falsche Dinge und beharrt darauf.

- Er kann sich nicht entscheiden.

Entsprechend sollte die Kommunikation individuell angepasst werden. D.h. Sie sollten immer im Blick haben, dass jeder demenziell erkrankte Mensch „seine Demenz" hat. Der Umgang ist also individuell! Die Kommunikation mit demenziell erkrankten Menschen ist durch mehrere Vorannahmen charakterisiert:

- Weisen Sie ihn nicht auf Fehler oder Defizite hin!

- Geben Sie vorsichtige Hilfestellungen bei Wortfindungsproblemen! 
- Vermeiden Sie Störreize!

- Verwenden Sie kurze einfache Sätze!

- Setzen Sie eine eindeutige Mimik und Gestik ein!

- Betonen Sie die Schlüsselwörter und setzen Sie diese ans Ende des Satzes!

- Halten Sie sich zum Gespräch im Blickfeld des Kranken auf!

- Bereiten Sie einen Themenwechsel langsam vor!

\section{Mögliche Probleme und praktische Tipps}

Unbefriedigte Bedürfnisse unterschiedlichster Art können zu Zuständen höchster Anspannung führen, wie z.B. Angst, Harndrang, Obstipation, Schmerzen, Durst und Hunger. Man sollte auch immer an einen entgleisten Diabetes denken! Die Kommunikation sollte mit einer höflichen Begrüßung und einer klaren und eindeutigen Vorstellung der eigenen Person beginnen.

Es kann von Vorteil sein, dass das Gespräch gemeinsam mit dem begleitenden Angehörigen oder Betreuer erfolgt. Dies muss aber nicht so sein. Es ist auch möglich, dass der Patient dies ablehnt, oder ihn dies sichtlich verunsichert. Dann sollte man nach Möglichkeit zuerst fremdanamnestisch tätig werden. Dabei kann man Anhaltspunkte erfahren, ob die Verhaltens- oder Wesensveränderungen akut sind!

Im Gespräch mit dem Patienten ist es ganz wichtig, sowohl sprachlich als auch mimisch und gestisch die mögliche innere Not des Patienten zu spiegeln und Hilfe anzubieten. Selbst wenn Sie die Gründe hierfür nicht erkennen können, begleiten Sie ihn solidarisch. Versuchen Sie, die Selbstoffenbarungsbotschaft zu ergründen. Versuchen Sie, sich in den Kranken „einzufühlen“, d.h. seine Selbstoffenbarungsbotschaften zu entschlüsseln. Anerkennen, akzeptieren und wertschätzen Sie die Selbstoffenbarungsbotschaften des Kranken, auch wenn sie unverständlich sind. Seien Sie neugierig und phantasievoll!

\section{Trial and error!}

Eine gute Beobachtung des Patienten ist in diesem Zusammenhang unerlässlich. Sie sollten neben der Mimik und Gestik insbesondere auf die Stimme, die Stimmung und die Haltung des Patienten achten und diese entsprechend aufnehmen und begleiten. Alles was Sicherheit beim Patienten erzeugen könnte, hat Bedeutung. Achten Sie immer auf die Reaktion des Patienten auf Ihr eigenes Agieren.

\section{Literaturempfehlung}

Brooker D (2008) Person-zentriert pflegen. Das VIPS-Modell zur Pflege und Betreuung von Menschen mit einer Demenz. Deutschsprachige Ausgabe hrsg. von Müller-Hergl C, Rüsing D, Verlag Hans Huber Bern

Cieplinska-Legge E, Kreisel S, Thomas C (2013) Delirdiagnostik und Sprachstörung. Vortrag auf dem 2. deutschsprachigen Delirtag Bielefeld 2013. URL: http://evkb.de/fileadmin/content/download/Psychiatrie/delirtag_ 2013/legge_workshop_delirdiagnostik_bei_patienten_mit_kommunikationsstoerungen_2013.pdf (abgerufen am 18.02.2015)

Gröning K (1997) Pflege in Zeiten der Fortschritts- und Konsumphilosophie. Dr. med. Mabuse 108(4), 29-36 
I Ärztliche Haltung im Alltag - praktische Tipps

Stiftung Wohlfahrtspflege NRW (Hrsg.) (2010) Demenzkranke Patienten im Krankenhaus - ein Praxishandbuch für Mitarbeiter in der Pflege. Schlütersche Verlagsgesellschaft Hannover 